\title{
Comparative genomics of pathogenic Leptospira interrogans serovar Canicola isolated from swine and human in Brazil
}

\author{
Luisa Z Moreno', Fabiana Miraglia², Frederico S Kremer ${ }^{3}$, Marcus R Eslabao ${ }^{3}$, \\ Odir A Dellagostin ${ }^{3}$, Walter Lilenbaum², Julio C Freitas ${ }^{4}$, Silvio A Vasconcellos ${ }^{1}$, \\ Marcos B Heinemann', Andrea M Moreno ${ }^{1 /+}$
}

\author{
'Universidade de São Paulo, Faculdade de Medicina Veterinária e Zootecnia, Laboratório de Epidemiologia Molecular e \\ Resistência a Antimicrobianos, São Paulo, SP, Brasil \\ ${ }^{2}$ Universidade Federal Fluminense, Departamento de Microbiologia e Parasitologia, Laboratório de Bacteriologia Veterinária, Niterói, RJ, Brasil \\ ${ }^{3}$ Universidade Federal de Pelotas, Centro de Desenvolvimento Tecnológico, Pelotas, RS, Brasil \\ ${ }^{4}$ Universidade Estadual de Londrina, Departamento de Medicina Veterinária Preventiva, Londrina, PR, Brasil
}

Leptospira interrogans serovar Canicola is one of the most important pathogenic serovars for the maintenance of urban leptospirosis. Even though it is considered highly adapted to dogs, serovar Canicola infection has already been described in other animals and even a few human cases. Here, we present the genomic characterisation of two Brazilian L. interrogans serovar Canicola strains isolated from slaughtered sows (L0-3 and L0-4) and their comparison with human strain Fiocruz LV133. It was observed that the porcine serovar Canicola strains present the genetic machinery to cause human infection and, therefore, represent a higher risk to public health. Both human and porcine serovar Canicola isolates also presented sequences with high identity to the Chinese serovar Canicola published plasmids pGuil and pGui2. The plasmids identification in the Brazilian and Chinese serovar Canicola strains suggest that extra-chromosomal elements are one more feature of this serovar that was previously unnoticed.

Key words: L. interrogans - serovar Canicola - genomics - plasmid.

Leptospirosis is a re-emerging zoonosis in both developed and developing countries (Dupouey et al. 2014). Leptospira interrogans is the most frequently reported species worldwide and of greater importance for public health (Haake \& Levett 2015, Wang et al. 2015). Many of L. interrogans serovars present importance for maintenance of urban leptospirosis and are described as adapted to biased reservoir hosts, such as serovar Copenhageni and rodents and serovar Canicola and dogs (Bolin 2000). Nevertheless, Hartskeerl and Terpstra (1996) already discussed the existence of variation of maintenance hosts and their respective serovars.

The L. interrogans serovar Canicola is one of the most important pathogenic leptospires and, although it is considered highly adapted to dogs (Schuller et al. 2015), serovar Canicola infection has already been described in swine and a few human cases (Lecour et al. 1989, Bolin 1994, Trevejo et al. 1998, Zhu et al. 2014). In Brazil, L. interrogans serovar Canicola has already been detected in wild and domestic dogs, cattle and swine (Freitas et al. 2004, Zacarias et al. 2008, Silva et al. 2014).

Here, we present the genomic characterisation of two Brazilian L. interrogans serovar Canicola strains (L0-3 and L0-4) isolated from slaughtered sows and their comparison with human strain Fiocruz LV133 (GenBank accession number AKWU2000000).

doi: 10.1590/0074-02760170119

Financial support: CNPq, CAPES, FAPESP (2011/18290-0, 2013/17136-2).

+ Corresponding author: morenoam@usp.br

Received 26 March 2017

Accepted 26 June 2017
The L0-3 and L0-4 strains were isolated from liver of slaughtered sows with unknown health history (Freitas et al. 2004), and later characterised as L. interrogans serovar Canicola (Miraglia et al. 2013). The strains were stored in Fletcher's medium (DIFCO/USA) enriched with $15 \%$ rabbit serum and maintained in EMJH broth (DIFCO/USA) at $30^{\circ} \mathrm{C}$, as part of the Leptospira collection of the Laboratory of Bacterial Zoonosis - University of São Paulo. The L0-4 strain is known to be virulent and commonly used in hamster virulence assays (Batista et al. 2010, Coelho et al. 2013).

Genomic DNA was extracted and purified with illustra $^{\mathrm{TM}}$ bacteria genomicPrep Mini Spin Kit (GE Healthcare do Brasil Ltda, São Paulo, Brazil). Whole-genome sequencing was performed through Illumina ${ }^{\mathrm{TM}}$ Miseq platform with 300 bp paired-end library. De novo assembly was performed with CLC Genomics Workbench 7.5.1 (CLC Bio, Denmark) and Geneious 10.0.3 (Biomatters Ltd, Auckland, New Zealand).

The obtained contigs were ordered according to $L$. interrogans serovar Copenhageni strain Fiocruz L1130 reference genome (GenBank accession numbers NC_005823 and NC_005824). Automatic genome annotation was performed with NCBI Prokaryotic Genome Annotation Pipeline (Tatusova et al. 2016). The basic assembly statistics and annotation features identified are summarised in Table.

L0-3 and L0-4 drafts genomes (GenBank accession numbers LIHE00000000 and LIIY00000000) present $\sim 4.2$ and 4.3 Mb for chromosome I and $\sim 400$ and $422 \mathrm{~kb}$ for chromosome II, respectively. BLASTn against nonredundant NCBI database was applied for the remaining contigs, that did not align with the reference, and resulted in high identity with Chinese serovar Canicola 
published plasmids pGuil (GenBank accession number NC 025136) and pGui2 (GenBank accession number NC_025197) (Zhu et al. 2014).

The same analysis was performed with strain Fiocruz LV133 available contigs; the draft genome of Fiocruz LV133 strain is available at NCBI database identified as L. interrogans serovar Canicola isolated from human in Brazil (GenBank accession number AKWU00000000). Fiocruz LV133 strain was characterised with $\sim 4.4 \mathrm{Mb}$ for chromosome I, $\sim 422 \mathrm{~kb}$ for chromosome II and also presented sequences similar to pGuil and pGui2.

Brazilian serovar Canicola drafts genomes were compared through Artemis comparison tool (ACT) (Carver et al. 2005) and BLAST ring image generator (BRIG) (Alikhan et al. 2011). Chromosomal content presented high synteny and $99 \%$ identity at DNA level among studied strains (Fig. 1). It is also worth noting a few deletion regions, in both chromosomes, in the serovar Canicola sequences compared to the reference; these correspond mainly to transposases, hypothetical and membrane proteins. These regions may be associated with serovar differences, considering that Nascimento et al. (2004) already described genome variations due to transposition of mobile genetic elements between $L$. interrogans serovars.
Both human and porcine serovar Canicola isolates present the major Leptospira virulence factors: lipoproteins LipL32 and LipL41; immunoglobulin-like factors LigA, LigB and LigC; flagelin; OmpA-like protein Loa22. These presented $100 \%$ identity with pathogenic L. interrogans serovar Copenhageni strain Fiocruz L1-130 genes. Therefore, the porcine serovar Canicola strains present the genetic machinery to cause human infection and represent a higher risk to public health.

The whole genome single nucleotide polymorphism (SNP) analysis was performed with CSI Phylogeny (Kaas et al. 2014) using L. interrogans serovar Copenhageni strain Fiocruz L1-130 as reference. For the phylogeny, the Maximum Likelihood method was used based on the general time reversible model. The SNP analysis, considering a pool of 24093 SNPs, enabled further differentiation between human and porcine serovar Canicola isolates despite their great similarity (Fig. 2). The porcine serovar Canicola isolates presented $96 \%$ of SNP identity distancing from the human serovar Canicola LV133 strain (91.5 to $92.6 \%$ identity).

Regarding plasmidial content, all sequences similar to the pGui plasmids present GC content $<34 \%$ differing from chromosomal content, as previously described

TABLE

Assembly statistics and basic annotation features observed for porcine Brazilian Leptospira interrogans serovar Canicola strains

\begin{tabular}{|c|c|c|c|c|c|c|c|c|c|}
\hline \multirow{2}{*}{ Strain } & \multirow{2}{*}{$\begin{array}{l}\text { GenBank } \\
\text { accession }\end{array}$} & \multicolumn{5}{|c|}{ Assembly statistics } & \multicolumn{3}{|c|}{ Basic annotation features } \\
\hline & & Scaffolds & $\mathrm{N}_{50}$ & Length & GC\% & Coverage & CDS & rRNAs & tRNAs \\
\hline L0-3 & LIHE00000000 & 319 & 24.742 & $4.71 \mathrm{Mb}$ & 35.2 & $40 x$ & 3,780 & 4 & 34 \\
\hline L0-4 & LIIY00000000 & 49 & 200.400 & $4.75 \mathrm{Mb}$ & 35.3 & $90 x$ & 3,899 & 5 & 37 \\
\hline
\end{tabular}

CDS: coding sequences; GC: guanine-cytosine content (\%).

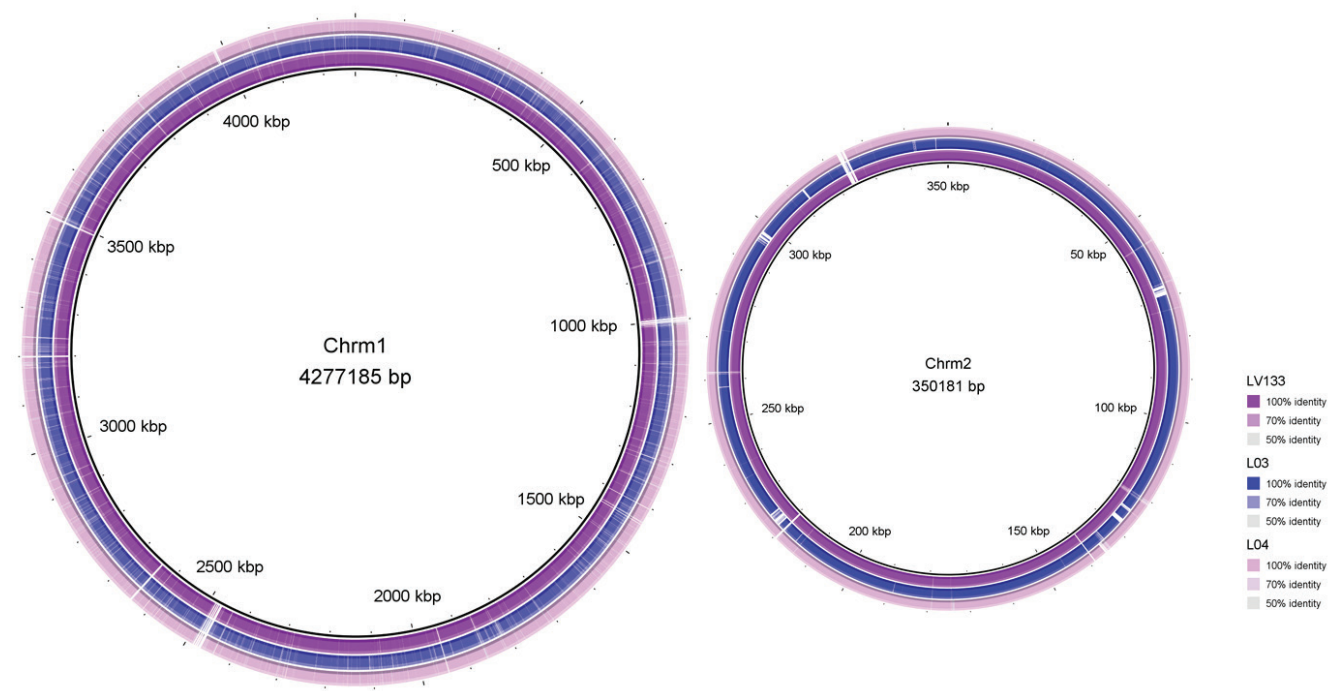

Fig. 1: BLAST ring image generator (BRIG) plot displaying whole genome comparison of Leptospira interrogans serovars Copenhageni (internal reference line) and Canicola. 
(Zhu et al. 2014). Despite the high similarity, the Brazilian pGuil homologous sequences present size variations (Fig. 3A). However, the main difference between serovar Canicola isolates is observed in the sequence similar to pGui2. Porcine isolates present plasmid sequences $\sim 11.2$ $\mathrm{kb}$ longer than the original pGui2 and LV133 plasmid (Fig. 3B), corresponding mostly to transposases and AAA ATPase domain proteins related to replication and recombination process.

The plasmid identification in the Brazilian serovar Canicola strains, as well as the Zhu et al. (2014) original description, suggest that extra-chromosomal elements are one more feature of this serovar that was previously unnoticed. The differences observed between plasmid

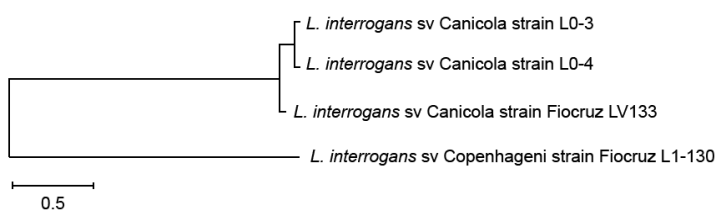

Fig. 2: single nucleotide polymorphism (SNP) analysis of Leptospira interrogans serovar Canicola human (LV133) and porcine (L0-3 and L0-4) isolates, using serovar Copenhageni strain Fiocruz L1-130 as reference.
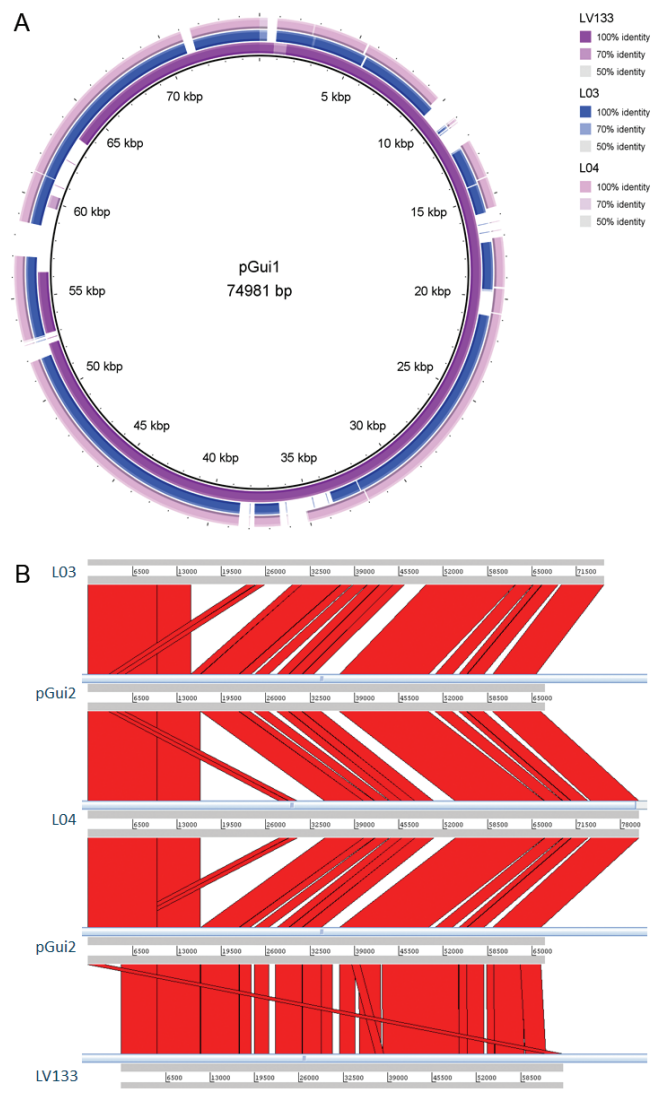

Fig. 3: comparative analysis of extrachromosomal sequences homologs to plasmids pGuil and pGui2. (A) Circular representation of the extrachromosomal element similar to pGuil. (B) Artemis comparison tool (ACT) synteny visualisation of extrachromosomal element similar to pGui2. sequences could be related to isolates origin (hosts and geographical) or even with the fact that L0-3 and L0-4 strains have been maintained in culture medium through multiple passages for the last ten years.

Considering that Lehmann et al. (2016) described $L$. interrogans serovar Lai in vitro culture-passage attenuation due to cumulative genome changes, mainly virulence genes mutations, the continued maintenance of the studied strains in EMJH medium could have significantly altered not only their chromosomal content, but also the plasmid sequences. Thus, L. interrogans serovar Canicola plasmids should be further studied to confirm if their differences are related to host specificities or environmental adaptions.

Nonetheless, the isolation of the serovar Canicola in pigs stands out in Brazil. Although serovars are not entirely unique to the host species, these usually present a certain preference (Schuller et al. 2015). Thus, the epidemiological implications of isolating serovar Canicola from pigs demands attention, with the possibility of Leptospira dissemination and maintenance on swine farms by dogs. The high similarity of $L$. interrogans serovar Canicola animal strains with the genome of the human strain also indicates that the porcine strains may pose a greater risk to public health than expected.

\section{AUTHORS' CONTRIBUTION}

LZM, SAV, OAD, WL and AMM - Conceived and designed the experiments; LZM and FM - performed the experiments; LZM and AMM - analysed the data; FSK, MRE and MBH - contributed with materials/bench work/analysis tools; JCF and SAV - samples collection and strains isolation; LZM, AMM, MBH, WL and OAD - contributed for the manuscript writing. All authors reviewed the manuscript.

\section{REFERENCES}

Alikhan NF, Petty NK, Ben Zakour NL, Beatson SA. BLAST Ring Image Generator (BRIG): simple prokaryote genome comparisons. BMC Genomics. 2011; 12(402): 1-10.

Batista CSA, Azevedo SS, Vasconcellos SA, Castro V, Figueiredo SM, Alves CJ, et al. Estabelecimento da leptospirose por infecção experimental em hamsters (Mesocricetus auratus) com Leptospira interrogans sorovar Canicola, estirpe LO4, por exposição cutânea íntegra e com abrasões. Braz J Vet Res Anim Sci. 2010; 47(5): $346-51$.

Bolin C. Leptospirosis. In: Brown C, Bolin C, editors. Emerging diseases of animals. Washington (DC): ASM Press; 2000. p. 185-200.

Bolin CA. Diagnosis of leptospirosis in swine. J Swine Health Prod. 1994; 2(3): 23-4.

Carver TJ, Rutherford KM, Berriman M, Rajandream MA, Barrell BG, Parkhill J. ACT: the artemis comparison tool. Bioinformatics. 2005 ; 21(16): 3422-3.

Coelho WA, Vasconcellos SA, Morais ZM, Ikuta CY, Gonçales AP, Souza GO, et al. Canine anti-leptospira bacterins commercialized in Brazil: a challenge made with indigenous strains of serovars Canicola and Copenhageni. Scientia Vitae. 2013; 1(1): 3-11.

Dupouey J, Faucher B, Edouard S, Richeta H, Kodjo A, Drancourt M, et al. Human leptospirosis: an emerging risk in Europe? Comp Immun Microbiol Infect Dis. 2014; 37(2): 77-83.

Freitas JC, Silva FG, Oliveira RC, Delbem ACB, Müller EE, Alves LA, et al. Isolation of Leptospira spp. from dogs, bovine and swine naturally infected. Cienc Rur. 2004; 34(3): 853-6. 
Haake DA, Levett PN. Leptospirosis in humans. Curr Top Microbiol Immunol. 2015; 387: 65-97.

Hartskeerl RA, Terpstra WJ. Leptospirosis in wild animals. Vet Quart. 1996; 18(S3): S149-50.

Kaas RS, Leekitcharoenphon P, Aarestrup FM, Lund O. Solving the problem of comparing whole bacterial genomes across different sequencing platforms. PLoS ONE. 2014; 9(8): e104984.

Lecour H, Miranda M, Magro C, Rocha A, Gonçalves V. Human leptospirosis - A Review of 50 cases. Infection. 1989; 17(1): 8-12.

Lehmann JS, Corey VC, Ricaldi JN, Vinetz JM, Winzeler EA, Matthias MA. Whole Genome shotgun sequencing shows selection on Leptospira regulatory proteins during in vitro culture attenuation. Am J Trop Med Hyg. 2016; 94(2): 302-13.

Miraglia F, de Morais ZM, Dellagostin OA, Seixas FK, Freitas JC, Zacarias FG, et al. Molecular and serological characterization of Leptospira interrogans serovar Canicola isolated from dogs, swine, and bovine in Brazil. Trop Anim Health Prod. 2013; 45(1): 117-21.

Nascimento AL, Ko AI, Martins EA, Monteiro-Vitorello CB, Ho PL, Haake DA, et al. Comparative genomics of two Leptospira interrogans serovars reveals novel insights into physiology and pathogenesis. J Bacteriol. 2004; 186(7): 2164-72.

Schuller S, Francey T, Hartmann K, Hugonnard M, Kohn B, Nally JE, et al. European consensus statement on leptospirosis in dogs and cats. J Small Anim Pract. 2015; 56(3): 159-79.

Silva FJ, Santos CEP, Silva GCP, Santos RF, Curci VCM, Mathias LA. The importance of Leptospira interrogans serovars icterohaemorrhagiae and Canicola in coastal zone and in southern fields of Rio Grande do Sul, Brazil. Pes Vet Bras. 2014; 34(1): 34-8.

Tatusova T, DiCuccio M, Badretdin A, Chetvernin V, Nawrocki EP, Zaslavsky L, et al. NCBI prokaryotic genome annotation pipeline. Nucleic Acids Res. 2016; 44(14): 6614-24.

Trevejo RT, Rigau-Pérez JG, Ashford DA, Mcclure EM, JarquínGonzález C, Amador JJ, et al. Epidemic leptospirosis associated with pulmonary hemorrhage - Nicaragua, 1995. J Infect Dis. 1998; 178(5): 1457-63.

Wang Y, Zhuang X, Zhong Y, Zhang C, Zhang Y, Zeng L, et al. Distribution of plasmids in distinct Leptospira pathogenic species. PLoS Neg1 Trop Dis. 2015; 9(11): e0004220.

Zacarias FGS, Vasconcellos SA, Anzai EK, Giraldi N, Freitas JC, Hartskeerl R. Isolation of Leptospira serovars Canicola and Copenhageni from cattle urine in the state of Paraná, Brazil. Braz J Microbiol. 2008; 39(4): 744-8.

Zhu WN, Huang LL, Zeng LB, Zhuang XR, Chen CY, Wang YZ, et al. Isolation and characterization of two novel plasmids from pathogenic Leptospira interrogans serogroup Canicola serovar Canicola strain Gui44. PLoS Negl Trop Dis. 2014; 8(8): e3103. 